\title{
Darwinian Selection Transforms Organisms to Bio-Robots
}

\section{Robert Skopec*}

Researcher-Analyst, Slovakia

Submission: April 23, 2018; Published: May 08, 2018

*Corresponding author: Robert Skopec, Researcher-Analyst, Dubnik, Slovakia, Email: zxcbnvm7@gmail.com.

\begin{abstract}
Marwin Minsky, one of the founders of artificial intelligence (AI) thinks that there may be tell-tale signs if programmer of our mass simulation has made some slips. He said that some laws of physics that aren't quite right could be the start of being able to prove that the Universe is a simulation. The illusory effect in the experiments started to fade if the brushstroke went higher than about 15 inches above the rubber hand. In other words, that's as far your Force Field goes. Scientific experiments can't grasp a fourth dimension any better than we can. Their studies can produce shadows of a sort, which suggest a fourth dimension really is there lurking just beyond our grasp. An illustration of clustering patterns due to Baryon Acoustic Oscillations (BAO), where the likelyhood of finding a galaxy at a certain distance from any other galaxy is governed by the relationship between dark matter and normal matter. If there is a new interaction between dark matter and radiation, the greatest cosmic controversy about the expanding Universe might be have an incredible resolution. Here we must as first formulate a new Main Natural Law: the Quantum Entanglement Entropy (QEE). Through above resonances the QEE is causing a metastasis of correlations, antagonistically intertwining (coincidences) all types of potentially conflicting interests.
\end{abstract}

Keywords: The predictive coding; Prospect-masking; New biology; Human predators; robots; The real Self.

\section{Introduction}

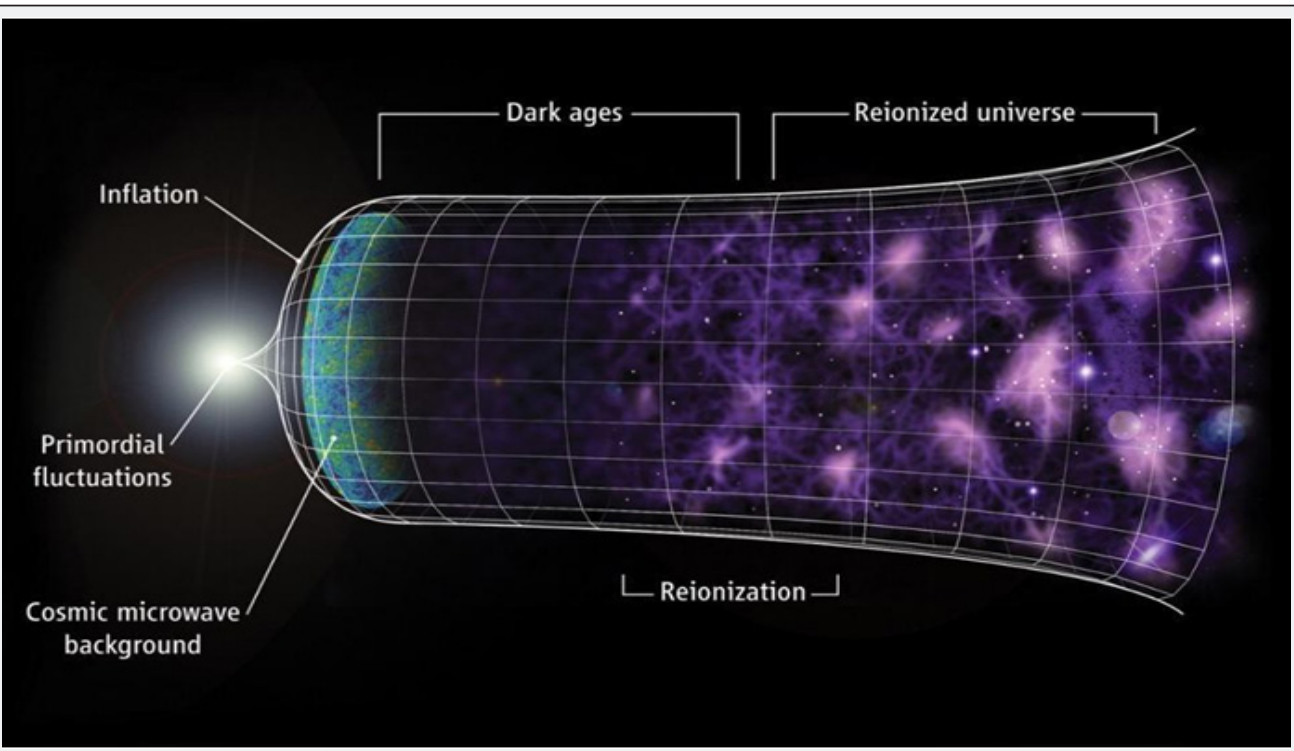

Figure 1: C. Faucher-Giguère, A. Lidz, and L. Hernquist, Science 319, 5859 (47)

Everything we know may just be part of a Matrix-style simulation, according to physicists who claim that we could all be part of a giant GAME. A new theory has suggested that our entire lives and memories may not be real, instead being part of a computer programme played by advanced robots. The so called simulation argument has been theorized for several years, with noted academics including Oxford University philosopher Nick
Bostrom, suggesting that the plot of the Matrix could be closer to real life than we think. Scientist say that we could all be living in the future, and our life in 2018 is nothing more than a series of numbers in a computer programme. It may sound like science fiction but researchers believe they may actually be able to prove that what you know isn't what you know. Marwin Minsky, one of the founders of artificial intelligence (AI) thinks that there 
may be tell-tale signs if programmer of our mass simulation has made some slips. He said that some laws of physics that aren't quite right could be the start of being able to prove that the Universe is a simulation. Silas Beane, from the University of Bonn, suggested several years ago that if humans were to build a small-sale simulation of the Universe we would be able to identify any constraints. These constraints would include a cut-off in the spectrum of high energy particles -exactly the kind of cut off in the energy of cosmic rays. This would be start of proving that our Universe is not what it seems - and that is part of a giant construct. These theories are not the first time that humans have debated whether we are actually real - French philosopher Rene Descartes theorized that nothing we perceive is true except our consciousness being aware of itself and its doubts. Thoughts can also be part of a simulation or programme that is being controlled partly by robots and partly by aliens. So if you're having a bad day today, it could be just some advanced robot in the year 2300 having a bit of fun with the character on his Play Station 29 (Figure 1).

\section{Humans have a force field around bodies}

Humans know their what their personal space is. Uninvited strangers invading it ivoke immediate sense of unease. It's like we don't end where our bodies end, but a little bit beyond. Now researchers have figured out a way to make you feel the invisible buble that wraps around you. In a study published in the journla Cognition, neuroscientists at the karolinska Institutet, Stockholm describe how they did this using an altered version of the so-called rubber hand illusion.
If both real and fake hands are simultaneously stroked by brush for a few minutes, the person's brain starts to accept the fake hand as part of the body. A few minutes later, the participants started to feel the rubber hand was their own. What's more, they started to sense what felt like a magnetic force or a force field between the brush and the rubber hand, according to study.

We can elicit this bizzarre sensation of there actually being something in mid-air between the brush and the rubber hand, Arvid Guterstam of the Karolinska Insitutet, a co-author of the study, told New Scientist. The finding plugs into research stretching to the 1990s that has suggested the brain has a mechanism to recognize the immediate space around the body, said neuroscientist Michael Graziano of Princeton University, who wasn't involved with the new study but has researched the topic.

The space around the body is processed like a jello mold. Like a thick layer of space around the body, deforming and moving as the limbs, M. Graziano told the Huffington Post. The illusory effect in the experiments started to fade if the brushstroke went higher than about 15 inches above the rubber hand. In other words, that's as far your Force Field goes.

Our brains contain a high-resolution map of our bodies. There are patches of neurons that respond to touch on your toes, their neighboring neurons respond to touch on your leg, and so on. But there are also groups of neurons in charge of recognizing the immediate space around the body, which is called the peripersonal space (PS) (Figure 2).

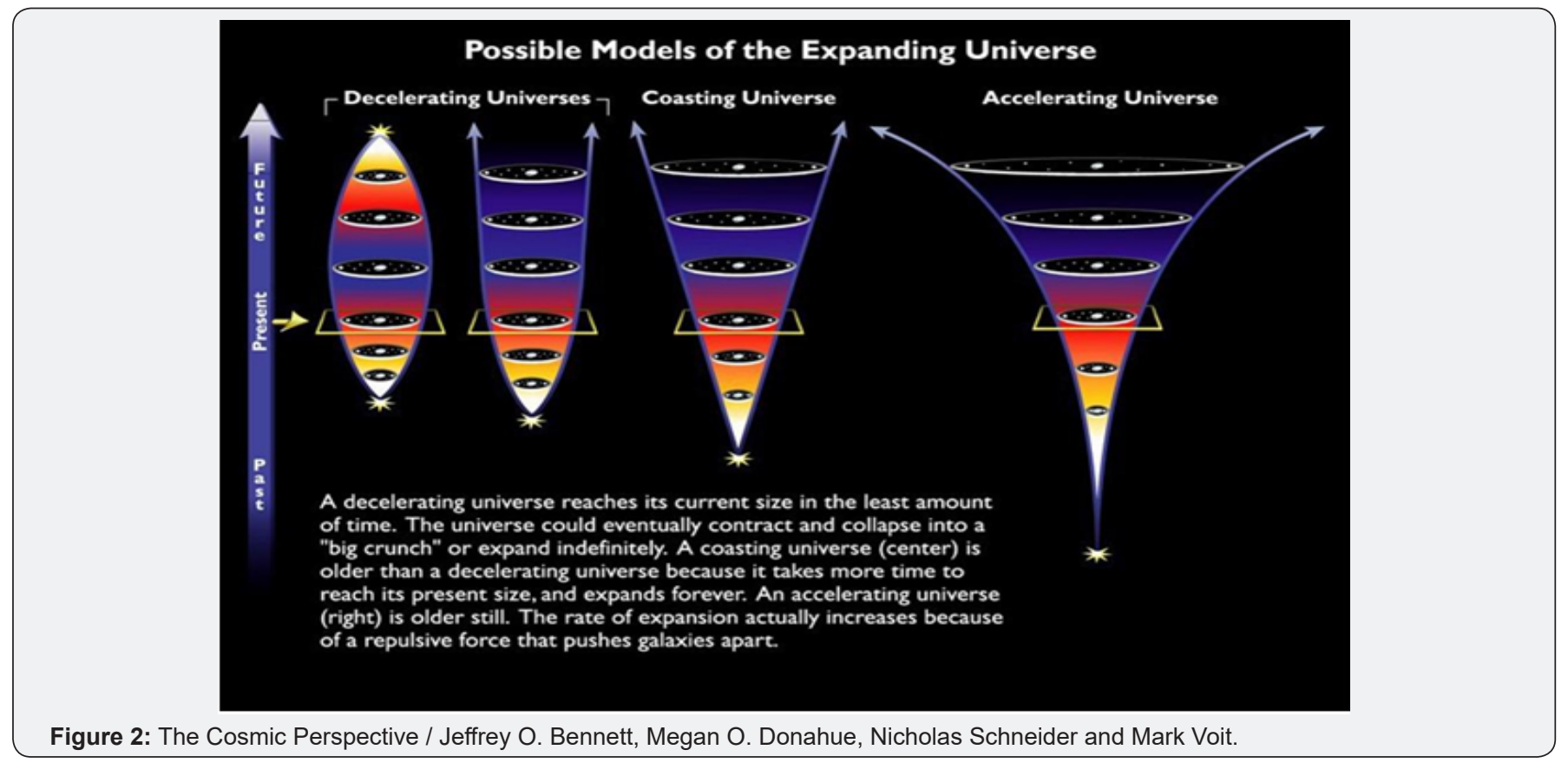

That PS space has several purposes, but its most important function is defense. This extended body representation in the brain may help us quickly detect approaching dangers. The discovery of PS goes back to animal studies conducted by M. Graziano and his colleaugues at Princeton University in early 1990s. They were trying to study visually responsive neurons in an obscure part of the brain - the claustrum - but they ended up finding more interestin signals in a nearby part of the brain, the basal gandlia. 
The researchers stumbled on neurons that responded to both touch and vision. The same neuron would become active if you touched the body in a specific area and when you moved a visible object into a region of space near the area.

At the same time, nobody knew the function of these neurons, M. Graziano said. It was not until his experiments in the early 2000s that they realized primarily emphasided the protection of the body. When they electrically stimulated those neurons, revving them up, the stimulation would induce a complex, coordinated behavior that resemled ducking or blocking. Then electrical stimulation of those neurons cause the subject to squint, turn the head toward the left, hunch the right shoulder and lift the right hand to a blocking posture near the face. This movement was so automatic that it could be evoked even under anesthesia.

We now understand these PS neurons are important in monitoring the margin of safety around the body, M. Graziano said. Most data on these neurons comes from monkeys, but brain scans of humans have revealed similar groups of neurons in our brains. Sometimes, people with certain types of brain damage lose their ability to process the peripersonal space. These people have no troble processing distant space, but lose a sense of peripersonal space, M. Graziano said. There's reason to think that our fluid force field is also extendable [1].

\section{Experiments with lasers and light reveal alternate reality of fourth dimension}

Try picturing a fourth dimension. Veer left for a second dimension, then just jump up for a third. Then drive of into a fourth dimension, somewhere, somehow, in a direction we don't have a word for because our minds can't grasp even the idea of it. That's a fourth dimension of space, about which two new studies yield some clues.

Scientific experiments can't grasp a fourth dimension any better than we can. Their studies can produce shadows of a sort, which suggest a fourth dimension really is there lurking just beyond our grasp. Two such physics experiments have done just that, according to two new papers published in the journal Nature.
Researchers used two different approaches to dance around the idea of a fourth dimension. They looked for a specific types of theorized "fingerprint" that is two-dimensional and therefore within our capabilities instead, as Gizmodo reports. In both cases, the experiments demonstrated phenomena that actually require just two dimensions.

The tools required to get those phenomena to manifest were anything but mundane. One experiment relied on lasers to trap individual atoms of a highly reactive element called rubidium in a square, like a cat carefully sitting between taped lines. The other experiment used a box full of special glass that manipulates waves of light, wiggling the furthest edge of a box and seeing how the effects rippled throughout the box.

Studies let researchers explore fourth dimension theories in new ways. The physicists on both these projects aren't just looking for a nice brain teaser. They want to stretch our knowledge and technology as far as possible. Even if we can't yet actually experience a fourth dimension ourselves, they think reaching for the fringes of it could offer valuable lessons that we can implement here in the 3-D world we live in.

Maybe we will come up with New Physics in the higherdimensional physics in lower dimensions, lead author on one of the papers, Mikael Rechtsman, a physicist at Penn State University, told Gizmodo. (Bartels, 2018)

\section{New dark matter physics}

There is an enormous controversy in astrophysics today over how quickly The Universe is expanding. One camp of scientists, the same camp that won the Nobel Prize for discovering dark energy, measured the expansion rate to be $73 \mathrm{~km} / \mathrm{s} / \mathrm{Mpc}$, with an uncertainty of only $2.4 \%$. But a second method, based on the leftover relics from the Big Bang, reveals an answer that's incompatibly lower at $67 \mathrm{~km} / \mathrm{s} / \mathrm{Mpc}$, with uncertainty of only $1 \%$. It's possible that one of the teams has an unidentified error that's causing this discrepancy, but independent checks have failed to show any cracks in either analysis. Instead, New Physics might be the culprit. If so, we just might have our first real clue to how dark matter might be detected (Figure 3).
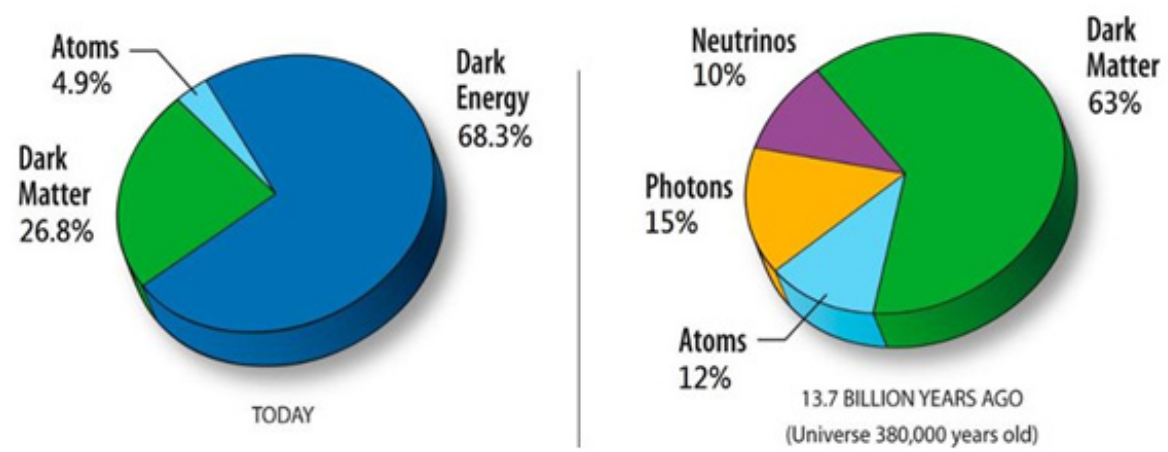

Figure 3: SA, modified by Wikimedia Commons user 老陳, modified further by E. Siegel. 
The expanding Universe has been one of the most important discoveries of the past 100 years, ans it's brought with it a revolution in how we conceive of the Universe. It was the key observation that led to the formulation of the Big bang, it allowed us to discover how stars and galaxies came to exist, it taught us the age of the Universe. Most recently, it led to the discovery of the accelerating Universe, whose cause we commonly call dark energy.

Dynamical dark energy, driven by some kind of field, that changes over time, could lead to differences in the Universe's expansion rate depending on when/how you measure it. General Relativity could be also wrong, and a modification to gravity might explain what appears to us an apparent acceleration. The evidence strongly points to that where dark energy is a cosmological constant.

The fluctuations in the Cosmic Microwave Background were first measured accurately by COBE in the 1990s, then moreaccurately by WMAP in the 2000s and Plack in the 2010s. This encodes a huge amount of information about the early Universe, including its composition, age and history.

But if you look at the early Universe, before there were stars and galaxies, all you had was the ionized plasma of normal matter, the hot mix of neutrinos and photons which acts as radiation, and the cold, slow-moving mass of dark matter. Based on physics of gravitation, trying to pull together matter and radiation.

In the new physics explanations could be a new type of interactions, either between dark matter and radiation, or by mixing in a new type of dark radiation. It's believed that the interactions between dark matter and radiation are understood, but the possibility that there are additional interactions, or a new type of radiation could change the picture.

If either photons, neutrinos, or some new type of dark radiation (that interacts with dark matter but not any of the normal particles). They might not only explain above cosmic controversy, and could be our fist hint of how dark matter might directly interact with other particles. It could even give us a clue to how to finally see dark matter directly.

An illustration of clustering patterns due to Baryon Acoustic Oscillations (BAO), where the likelyhood of finding a galaxy at a certain distance from any other galaxy is governed by the relationship between dark matter and normal matter. If there is a new interaction between dark matter and radiation, the greatest cosmic controversy about the expanding Universe might be have an incredible resolution.

Because there's new physics afoot is still undetermined, but it's vital to remain open-minded to these possibilities. The resolution to this paradox may be new physics, after all. And if it is, it just might teach us someting about the dark side of the Universe [2-4]. The evidence, from everything we've gathered, strongly points to that first case, where dark energy is a cosmological constant.

\section{Why the universe shouldn't exist at all}

Don Lincoln, a senior physicist at Fermilab, does research using the Large Hadron Collider. He is the author of "The Large hadron Collider: The Extraordinary Story of the Higgs Boson and Other Stuff That Will Blow Your Mind", and produces a series of science education videos. To the question: Why is there (in our Universe) something, rather than nothing ? He is giving the simplest scientific answer: We shouldn't exist at all.

Give some scientists 65 pounds of rare isotope of germanium, cool it to temperatures cold enough to liquefy air, and place their equipment nearly a mile underground in an abandoned gold mine, and you'll have the beginnings of an answer. Their project is called the Majorana Demonstrator and it is located at the Sanford Underground Research Facility, near Lead, South Dakota.

To grasp why science has trouble explaining why matter exists - and to understand the scientific achievement of Majorana - we must first know a few simple things. First, our Universe is made exclusively of matter, all people, the Earth, even distant galaxies. All of it is matter.

Our best theory for explaining the behavior of the matter and energy of the Universe contradicts the realities that we observe in the Universe all around us. This theory, called the Standard Model, says that the matter of the Universe should be accompanied by an identical amount of antimatter, which, as its name suggests, is a substance antagonistic to matter. Combine equal amounts of matter and antimatter and it will convert into energy. And the street goes both ways: Enough energy can convert into matter and antimatter (since antimatter's discovery in 1931).

Modern cosmology says the Universe began in an unimaginable Big Bang - an explosion of energy. In this theory, equal amounts of matter and antimatter should have resulted. So how our Universe made exclusively of matter ? However, we don't know the process whereby the asymmetry in the laws of the Universe arose. One possible explanation revolves around a class of subatomic particles called leptons. The most well-known of the leptons is the familiar electron, found around atoms. A less known lepton is called the neutrino. Neutrinos are emitted in a particular kind of nuclear radiation, called beta decay. It occurs when a neutron in an atom decays into a proton, an electron, and a neutrino. Neutrinos are fascinating particles. They interact extremely weakly, a steady barrage of neutrinos from the nuclear reactions in the Sun pass through the entire Earth essentially without interacting.

Still a mystery to scientists is whether there is a difference between neutrino matter and neutrino antimatter. While we know that both exists, we don't know if they are different 
subatomic particles or if they are the same thing. We don't know which kind of twins the neutrino matter/antimatter pair are. If neutrinos are their own antimatter particle, it would be an enormous clue in the mistery of missing antimatter.

The way they do that is to look first for a very rare form of beta decay, called double beta decay. That's when two neutrons in the nucleus of an atom simultaneously decay. If neutrinos are their own antiparticle, an even rarer thing can occur called neutrinoless double beta decay. In this process, the neutrinos are absorbed before they get outside of the nucleus. In this case no neutrinos are emitted. The observation of a single, unambiguous neutrinoless double beta decay would show that matter and antimatter neutrinos were the same.

If indeed neutrinoless double beta decay exists, it's very hard to detect and it's important that scientists can discriminate between the many types of radioactive decay that mimic that of a neutrino. This requires the design and construction of very precise detectors. So that's what the Majorana Demonstrator scientists achieved. Once and for all, it can answer the question of whether matter and antimatter neutrinos are the same or different. With that information in hand, it might be possible to understand why our Universe is made of matter [6].

\section{How the majorana fermion might change the world}

Chinese scientists won a major victory, by proving that the Majorana fermion - a particle we've found tantalizing hints of for years - genuinely exists. This discovery has huge implications for quantum computing, and it might change the World.

A Majorana fermion is weird even by the standards of quantum physics. The Majorana fermion doesn't have a charge, which allows it to be matter and anti-matter at the same time! The fact that it doesn't have a charge, and also happens to be the exact reverse of itself at the same time.

Quantum computers, are like a huge pile of dimmer switches. You can set these dimmers much, much faster than you can flip on this light switches, because the dimmers are all wired to each others, immediately. These dimmers, e. i. quantum bits, are what's called entangled. If you change one quantum bit, the others it's entangled with change with it, even if they're a million miles away from each other. That's where Majorana fermions come in due to their no-charge nature [7].

\section{Dichotomous correlations of masking}

In medicine, translational research is increasingly a separate research field. A citation pattern between the applied and basic sides in cancer research appeared around 2000. Given the our previous observations concerning patients with first episode psychosis show altered neural responses to prediction error (PE). This alteration in turn relates to the severity of their delusional ideation, results provide novel evidence for view that schizotypy relates to psychosis at more than just a superficial descriptive level. Healthy unusual ideas have their source in aberrant striatal functioning. They may find this personally, socially and even financially advantageous. A striatal system capable of entertaining irrelevant associations is not detrimental given the link between creativity and healthy schizotypy (generate novel or unusual associations). A deficit in belief evaluation is associated with right frontal cortex dysfunction.

In blocking prior learning leads to an attenuation of new learning because of subsequently reduced expectation that a blocked stimulus has a predictive power. An inappropriate dorsolateral prefrontal (DLPFC) PE signal during causal learning in subjects with psychosis was predictive of the severity of delusions. It's an evidence that aberrant right frontal PE signal relates to schizotypy. The severity of subject's baseline magical ideation is correlated negatively with magnitude of their striatal PE response to the blocked cue. People with highest degrees of distress showed least PE response to violation of blocking-induced expectation in frontal cortex. Subjects with inappropriate DLPFC response during blocking trials were most distressed by their odd beliefs and not the reality [8].

\section{Neurobiological correlates of prospect-masking}

Translational medicine is a rapidly growing discipline in biomedical research and aims to expedite the discovery of new diagnostic tools and treatments by using a multi-disciplinary, highly collaborative, "bench-to-bedside" approach. The question is if our experience of reality is accurate or is a fiction ? For these fascinating processes F. Crick and $\mathrm{Ch}$. Koch coined a term: neuronal correlates of consciousness (NCCs). Given that the processing time of lone neurons is in the millisecond range, this categorization is remarkably swift and can be accomplished only via massive parallel processing. Our perceptions lag behind reality, casting doubt on our presumed unity of consciousness. For example, when stimuli follow in rapid succession, registering one image can distort previous or subsequent images or suppress them completely. Psychologists refer to this effect as masking.

Masking makes it clear that our perception can deviate significantly from reality. Such systematic distortions of perception teach us the rules that the mind uses to construct its view of the world. The most used technique is backward masking, in which the mask follows an initial stimulus. Both stimuli can fuse completely. Two images in rapid succession result in a single conscious impression. Masking can eliminate conscious recognition, while only the input stages of the visual brain were activated. This means that even an image that strikes the retina one tenth of a second after a prior image can cancel out conscious perception of the first image. The masking thwarts the development of a visual impression, it cannot prevent unconscious processing: it had been masked from conscious perception [9].

As soon as neuronal signals within the visual cortex or between the cortex and deeper brain regions start shuttling back and forth, as they do, subsequent information can distort 
the processing of earlier information. Our perceptions lag considerably behind reality - and we don't notice that. In all probability, subsequent brain activity during backward masking disturbs precisely those processes that signal the onset and disappearance of a target stimulus. This competition among overlapping neural coalitions are a significant feature of the censorship in a consciousness.

If sensing such a simple input can be so variable, imagine how complicated it must be for the brain to assess the actual world. How do we integrate all these details into a unified image that conveys a person's identity, gender and emotional state ? This question goes to the core of the so-called binding problem. If NCCs arise within the various processing centers in the brain at different times, shouldn't each of the attributes be perceived with a time lag ? The answer may be given by the mechanism of Dependence Length Minimisation (DLM) [10]. A unified impression is rapidly reached because the brain has no mechanism for registering the asynchrony [11].

We simply perceive all the qualities of an object simultaneously - as incoherent as that composite image might be. It means that continuity of consciousness may be yet another illusion. Consider patients who experience "cinematographic vision". They lose their sense of visual continuity and instead see a flickering series of still images. The images do not overlap or seem superimposed, they just last too long, like a movie that has been stuck on freeze frame and then suddenly jumps ahead to catch up to a real-time moving scene.

Our perception seems to be the result of a sequence of individual snapshots, a sequence of moments, like individual, discrete movie frames that quickly scrolling past us, we experience as continuous motion. We experience events at the same moment as synchronous, that reach us sequentially and are perceived in that order. The duration of such snapshots is between $20-200$ milliseconds. If the individual images were shorter in duration - there were more of them per unit of time - then time would appear to pass more slowly. People who have been in automobile accidents, natural catastrophes and other traumatic events often report that at the height of drama, everything seemed to go in slow motion ("time dilation"). It is a question of how the brain mediates our sense of time. In fact, changing coalitions of larger neuron groups are the neuronal correlates of consciousness which is generating our flow of consciousness [12].

\section{Accomodation as deceiving others}

The term "dichotomous" we are using as meaning of "twofaced", e.i. as something that has it's good face and also it's bad face at the same time. Between basic authors on the topics of the masking we can name psychiatrist R. D. Laing with his first book titled The Divided Self. The dilemma of the divided self is that the more isolated "the real self" becomes, the less equipped it is to contend with reality and ordinary developmental processes. Moreover, "the false self" becomes increasingly identified with the publicly observable body, and the real self with person's invisible mind. As mind and body become more split, the real self becomes more volatile, disembodied, accommodating and/or deceiving others. This schizoid and psychotic potential than can be used during masking unfairly for one's own advantage [13].

\section{Default-mode network}

In the hierarchical architecture a secondary process provides top-down predictions to reduce free-energy associated with the Freudian primary process, which is converting free energy into bound energy. In both accounts, higher cortical areas are trying to organize activity in lower-levels through suppression of their energy. The so called default-mode network (DMN) is a network of regions that show high metabolic activity (glucose metabolism, oxygen consumption) and blood flow at rest but which deactivate during goal-directed cognition [14].

The medial prefrontal cortex sends dense projections to the ventral striatum and midbrain. The ventral striatum displays functional connectivity with the midbrain, medial temporal lobes and higher-level nodes of the DMN and the midbrain and ventral striatum signal prediction error and motivationalsalience. The attention system is activated during externallydirected cognition and deactivated during internally-directed cognition, while the opposite is true of the DMN. Much of the brain's vast energy budget is reserved for spontaneous neuronal activity. Spontaneous activity in the DMN reflects the constant containment of spontaneous endogenous activity. The spontaneous activity in the dorsal attention system indexes the continual monitoring and suppression of exogenous stimuli [15].

Based on empirical findings it was proposed that bursts of limbic theta recorded in the cortex as increased gamma may index the positive symptoms of various neurologic and psychiatric disorders. These findings imply that theta modulates coupling between theta and gamma and theta can promote longrange coupling in cortical networks. A retreat from the external world is characteristic of a loss of an intense object-love. The patient reacts to this loss by targeting the aggression felt the lost object back upon his/her own ego. A reduced task-induced suppression of DMN activity is related to reduced blood flow and activation in the dorsolateral prefrontal cortex, hyper-perfusion, metabolism and activity in limbic and medial prefrontal regions.

\section{Results}

\section{Human creativity means anti-predatory}

Within public health, translational medicine is focused on ensuring that proven strategies for disease treatment and prevention are actually implemented within the community. When a scene hits the cones (color receptors) in the eye, neural signals from the retina travel to the area 17 , in the occipital lobe at the back of the brain. There the image is processed further within local clusters. Afterward, the information is sent forward and distributed to several regions in the temporal and parietal lobes. In the case of color, the information goes to area V4 in 
the fusiform gyrus of the temporal lobe. From there it travels to areas farther up in the hierarchy of color centers, including a region near a patch of cortex called the TPO (for the junctions of the temporal, parietal and occipital lobes).

Abstract, numerical computations happen in stages. An early step also take place in the fusiform gyrus, where the actual shapes of numbers are represented. Later one occurs in the angular gyrus, a part of the TPO concerned with numerical concepts (ordinality: sequence, cardinality: quantity). Brainimaging studies in humans suggests that visually presented letters or numbers activate cells in the fusiform gyrus, while the sounds are processed higher up in the TPO. The numbercolor synesthesia is caused by cross-wiring between V4 and the number appearance area (both within the fusiform) or between the higher color area and the number-concept area (both in the TP0). (Ramachandran and Hubbard, 2001). The processes of cross-linking in TPO is showing its potentially important role during generating the level of human creativity and geniality [16].

The neurological basis of synesthesia could help explain one skill that many creative people share facility for using metaphor to make links between seemingly unrelated domains. Metaphor involves making links between seemingly unrelated conceptual realms, while this is not just a coincidence. Coincidence is at a deeper level a manifestation of entanglement entropy [17]. Mutation of the angular gyrus make possible for synesthesia to provide excess communication among different brain maps. Involved in cross-modal synthesis the brain regions where information from touch, hearing and vision flow together are enabling the construction of high-level perceptions. The angular gyrus is disproportionately larger in humans than in apes and monkeys - evolved originally for cross-modal associations. Probably later became co-opted for more abstract functions such as metaphors [18].

The common abstract property is extracted somewhere in the vicinity of the TPO, probably in the angular gyrus. It is extracting the abstraction of the common denominator ("ratio") from a set of strikingly dissimilar entities. When the ability to engage in cross-modal abstraction emerged, it is opening the way for more complex types of abstraction - e. i. for geniality of thinking [19].

\section{Nonlinear deviation term}

Neurobiological correlates of value have been described in orbitofrontal (conscience), cingulated cortex (critical intellectuals) and the basal ganglia, areas of the brain traditionally associated with reward-seeking behavior.

Some neurons in orbitofrontal cortex represent value independently from evidence, choice and action. Anterior cingulate cortex is thought to represent negative (critical, nonlinear) value [20,21].
There is much evidence that a number of brain regions are sensitive to expected reward (or "utility"). The most well established are dopaminergic regions such as the striatum and midbrain structures. The common ratio pattern can be reconciled by the plausible assumption that people apply nonlinear decision weights $\pi(p)$ to objective probabilities $p$, so that the ratio $\pi(0.02) / \pi(0.01)$ is much smaller than $\pi(1) / \pi(0.5)$.

Neural responses to probabilities resembling the smoothly increasing function which typically fit behavior well. Paulus and Franck (2006) focused on between subjects measures and showed that activity in anterior cingulate correlated with degree of nonlinearity across subjects. We can make the assumption that neural activity is approximately a linear function of the behaviorally derived utility function. The GLM model separates the weighting function into two components: (1) component that is linear in $p$ and (2) the component that is the nonlinear deviation term (NDT) $\Delta\left(p, \alpha_{i}\right)=\pi\left(p, \alpha_{i}\right)-p$.

Specifically, we are looking for a prospect-theoretic expected value function that is nonlinear in $p$; that is $\pi(p, \alpha) u(x)=p \cdot u(x)+\Delta(p, \alpha) \cdot u(x)$. We assume the function $(x)$ is power function $x^{p}$, where the value of $p$ is taken from the individual behavioral estimate, and $\Delta\left(p, \alpha_{i}\right)=\pi\left(p, \alpha_{i}\right)-p$, where the mean group $=0.771$ is used.

If the expected utility (EU) null hypothesis is an accurate approximation of valuation of risky choices, there should be no reward-related brain regions that respond to the deviation term $\Delta(p, \alpha) \cdot u(x)$. If the nonlinear weighting hypothesis is an accurate approximation, there should be reward-related brain regions that respond equally strongly to the linear component $p \cdot u(x)$ and to the nonlinear component $\Delta(p, \alpha) \cdot u(x)$.

We can test whether cross-subject variation in the inflection of nonlinear weighting inferred from choices is consistent with cross-subject differences in neural activity. More highly nonlinear functions will be approximated by a combination of the linear term $p$ and the nonlinear term $\Delta\left(p, \alpha_{i}\right)=\pi\left(p, \alpha_{i}\right)-p$ that puts more weight on the nonlinear term. A linear-weighting subject, will put no weight on nonlinear deviation $\Delta\left(p, \alpha_{i}\right)=\pi\left(p, \alpha_{i}\right)-p$.

Denote the true weighting function for subject $i$ by $\pi\left(p, \alpha_{i}\right)$ , and the deviation from linear weighting by $\Delta\left(p, \alpha_{i}\right)=\pi\left(p, \alpha_{i}\right)-p$ . A brain region that represents $\pi\left(p, \alpha_{i}\right)$ will be significantly correlated with both $\Delta\left(p, \alpha_{i}\right)$ and $p$.

That is, the linear term and nonlinear deviation term with a higher weight on the nonlinear deviation term [22]. Brain regions that are significantly correlated with the nonlinear term include the anterior cingulated cortex (ACC), the striatum, motor cortex, and cerebellum. Our intuition is that brain activity during valuation of risks is more likely to correspond to cognitive components of prospect-masking, than to EU, and it will be easier to construct an adaptationist account of how evolution 
would have shaped brains to follow prospect-masking rather than EU. The prospect-masking follows from psychophysics, while EU from normative logic [23].

The increased specialization required today for professional credentials makes the broad thinking of that characterizes geniuses harder to develop. I agree that the ritual culture of academia may also hamper genius. As philosopher of science Thomas Kuhn has pointed out, highly creative work (without precedent) does not fit existing formalistic academic paradigms tend to be dismissed (the counter-selection) [24]. Many great scientists have related how their most original ideas were repeatedly rejected by their peers.

\section{Dichotomous correlations of adaptation}

One prevalent description of translational medicine, first introduced by the Institute of Medicine's Clinical Research Roundtable, highlights two roadblocks (i.e., distinct areas in need of improvement): the first translational block (T1) prevents basic research findings from being tested in a clinical setting; the second translational block (T2) prevents proven interventions from becoming standard practice.

An important role in the processes of adaptation and masking in humans is playing also the immune system. The innate immune system functions as an interpreter of tissue damage and provides a first line of defense, also translates the information to other repair and defense systems in the body by stimulating angiogenesis, wound repair, and activating adaptive immunity. It is appropriate to consider autophagy a means for programmed cell survival balancing and counter-regulating apoptosis. Autophagy seems to have a dichotomous role in tumorigenesis and tumor progression.

Two other attributes play a similarly paradox role. The first involves major reprogramming of cellular energy metabolism in order to support continuous cell growth and proliferation replacing the metabolic program that operates in most normal tissues. The second involves active evasion by cancer cells from attack and elimination by immune cells. This capability highlights the dichotomous correlations of an immune system that both antagonizes and enhances tumor development and progression. Evidence began to accumulate in the late 1990s confirming that the infiltration of neoplastic tissues by cells of the immune system serves counter-intuitively to promote tumor progression $[25,26]$.

\section{The twofaced new main law of Nature}

The quantum entanglement is a basis of twofaced reality in which we are living our lives. From this reality are outgoing also the science and healthcare too. Altough metastasis is important for systemic correlations expansion (as in tumors), it is a highly dichotomous process, with millions of cells being required to disseminate to allow for the selection of cells-correlates aggressive enough to survive the metastatic cascade. To quantify the dynamics of metastasis of correlations development, we need look at the coincidences of metastases in terms of co-occurrence at every point of time. To quantify co-occurrence we can use the -correlation between dichotomous variables defined as:

$$
\frac{N_{X}(t) C_{i j}(t)-m_{i}(t) m_{j}(t)}{\sqrt{m_{i}(t) m_{j}(t)\left[N_{X}(t)-m_{i}(t)\right]\left[N_{X}(t)-m_{j}(t)\right]}}
$$

where $C_{i j}(t)$ is the number of co-occurrence at time t. Than $\mathrm{i}$ and $\mathrm{j}$ represent particular site of metastasis, $\mathrm{X}$ represents the primary correlations type. The pair-wise correlations (coincidences) between metastasis network links for every primary correlations types and lead to the correlation coefficient matrix.

The dichotomous correlations of the adaptation may be caused also by the Quantum Entanglement Relative Entropy as a measure of distinguishability between two quantum states in the same Hilbert space. The relative entropy of two density matrices $p_{0}$ and $p_{1}$ is defined as $S\left(p_{1} \mid p_{0}\right)=\operatorname{tr}\left(p_{1} \log p_{1}\right)-\operatorname{tr}\left(p_{1} \log p_{0}\right)$. When $p_{0}$ and $p_{1}$ are reduced density matrices on a spatial domain $\mathrm{D}$ for two states of a quantum field theory (QFT), implies that $S\left(p_{1} \mid p_{0}\right)$ increases with the size of D. Than $\Delta S_{E E}=-\operatorname{tr}\left(p_{1} \log p_{1}\right)+\operatorname{tr}\left(p_{0} \log p_{0}\right)$ is the change in entanglement entropy across $\mathrm{D}$ as one goes between the states.

When the states under comparison are close, the positivity is saturated to leading order: $S\left(p_{1} \mid p_{0}\right)=\Delta\left\langle H_{\text {mod }}\right\rangle-\Delta S_{E E}=0$. (Skopec II., 2018)

The problem of conventional adaptation may be given by a definition of static, deterministic world. The proliferative correlations lead to the resonances between the degrees of freedom. When we increase the value of energy, we increase the regions where randomness prevails. For some critical value of energy, chaos appears: over time we observe the exponential divergence of neighboring trajectories. For fully developed chaos, the cloud of points generated by a trajectory leads to diffusion. (Prigogine, 1997) Here we must as first formulate a new Main Natural Law: the Quantum Entanglement Entropy (QEE). (Skopec III., 2015) Through above resonances the QEE is causing a metastasis of correlations, antagonistically intertwining (coincidences) all types of potentially conflicting interests.

\section{Focus on cross-functional collaborations}

Another masked problem of dichotomous correlations in cancer arose from conflicting effects of E-cadherin and p120, adhesion proteins that are essential for normal epithelial tissues to form, and which have long been considered to be tumor suppressors. New study has found that this hypothesis didn't seem to be true, since both E-cadherin and p120 are still present in tumor cells and required for their progression. That led researchers to believe that these molecules have two faces: a good one, maintaining the normal behavior of the cells, and a 
bad one, that drives tumorigenesis. It uncovers a new strategy for cancer therapy [27]. This finding represents an unexpected New Biology that provides the code, the software for turning off cancer.

An another new research estimates that ocean fishing has resulted in humans exploiting adult fish populations at about 14 times the rate of other marine predators, while humans have hunted and killed adult land animals at round nine times the rate of other animal predators [28].

Human hunting and fishing has had an extraordinary impact on the natural world and its ruthless efficiency is laid bare in detailed survey of 2, 125 species of terrestrial and marine predators around the world. The study revealed that human hunting and fishing is qualitatively different to the predatory behavior shown by other species. It has, concentrated on killing mature adult animals rather than their offspring, which the scientists have likened to eating into the reproductive capital rather than the reproductive interest of the natural world.

Whereas predators primarily target the juveniles e. i. reproductive interest of populations, humans draw down the reproductive capital e. i. exploiting adult prey. The study found that humans have fundamentally changed the balance of marine ecosystems [29].

Our wickedly efficient killing technology, global economic systems, and resource management that prioritise short-term benefits to humanity have given rise to the human superpredator. Our impacts are as extreme as our behavior and the planet Earth bears the burden of our predatory dominance. In fact, the sustainable exploitation paradigm management is typical for all global activities of humans. Humans by above overexploitation have altered course of evolution.

But what is masking this super-predatory behavior of humans? Brain mechanisms involved in predatory aggression activated in violent intra- and extra-specific aggression are very similar. Unemotional violence associated with antisocial personality disorder is called predatory because it involves restricted intention signaling and low emotional/physiological arousal, including decreased glucocorticoid production. This epithet is covering a structural similarity at the level of the hypothalamus where the control of affective and predatory aggressions diverges.

Aggressive encounters activate the mediobasal hypothalamus, a region involved in intra-specific aggression. The activation of the lateral hypothalamus is involved in predatory aggression. Glucocorticoid deficiency increased activation in the central amygdala, also involved in predatory aggression. In adition, activation patterns in the periaqueductal gray - involved in autonomic control - is also seen in predatory aggression. The above findings suggest that antisocial and predatory aggression are not only similar, but are controlled by overlapping neural mechanisms.

\section{Neural and evolutionary basis of prospect-masking}

Establishing a neural and evolutionary basis of prospectmasking can show how the foundation for principles guiding social science might be usefully shifted from relying largely on logic, to respecting biological implementation. It may include convergence to logical principles as a result of learning or higher-order cognition.

So called prior probability affects the threshold for initiating an action. Priors can be manipulated by changing the probability of the target which is shown to the right or left fixation. Differences in the distribution of reaction time for different priors conformed to the "swivel" prediction, suggesting a change in the threshold and in the rate of rise of decision variable (DV). The threshold appeared to change as a linear function of the logarithm of the prior, which is suggesting that the DV has units $\log (\mathrm{P})$. This implies a form of probabilistic reasoning, with the DV representing a level of certainty that the prepared movement should be executed.

Further studies have shown that different decision strategies that favor urgency, certainty, or prior expectations seem to trade off in these units of $\log (\mathrm{P})[30]$.

Even more important, there exists a multiplicity of evolutions, which are particularly evident in the field of biology. As stated by Stephen J. Gould, bacteria have remained basically the same since the Precambrian era, while other species have evolved dramatically, often over short time scales. It would therefore be a mistake to consider a simple one-dimensional evolution [31]. Some two hundred million years ago, certain reptiles started to fly, while others remained on earth. At a later stage, certain mammals returned to the sea, while others remained on land. Similarly, certain apes evolved into humanoids, while others did not.

At the conclusion it is appropriate to cite Gould's definition of the historical character of life: To understand the events and generalities of life's pathway, we must go beyond principles of evolutionary theory to a paleontological examination of the contingent pattern of life's history on our plane - the single actualized version among millions of plausible alternatives that happened not to occur.

Such a view of life's history is highly contrary both to conventional deterministic models of Western science and to the deepest social traditions and psychological hopes of Western cultures for a history culminating in humans as life's highest expression and intended planetary steward [32].

Humans are a unique super-predator that hunts and kills other species many times more efficiently tha other top predators both on land and sea. Masking means that what you know isn't what you know. A new theory has suggested that our entire lives and memories are maybe not real, instead being part of a computer programme played by advanced bio- robots. 


\section{Darwinian selection process promotes spreading and forming distant tumors}

Cancer metastasis, the migration of cells from a primary tumor to form distant tumors in the organism, can be triggered by a chronic leakage of DNA within tumor cells, according to a team led by Weil Cornell Medicine and Memorial Sloan Kettering Cancer Center researchers.

How metastasis occurs has been one of the central mysteries of cancer biology. The findings, published in Nature, appear to have partly solved this mystery. The authors traced the complex chain of events that results from chromosomal instability- a widespread feature of cancer cells in which DNA is copied incorrectly every times these cells divide, resulting in daughter cells with unequal DNA content. Using models of breast and lung cancer, the investigators found that chromosomal instability leads to changes in the organisms that drive metastasis. They showed that chromosomal instability can cause a leakage of DNA from the nuclei of cancer cells, leading to a chronic inflammatory response within the cells. The cells essentially can hijack that response to enable themselves to spread to distant organs, said study lead author Dr. Samuel Bakhoum, a Holman research fellow at Weill Cornell Medicine and a senior resident in radiation oncology at Memorial Sloan Kettering Cancer Center.

The discovery is principally a basic science advance, but can also have long-range implications for cancer drug development. Metastasis cause 90 percent of cancer deaths, and this work opens up new possibilities for therapeutically targeting it, said senior author Dr. Lewis Cantley, the Meyer Director of the Sandra and Edward Meyer Cancer Center and a professor of cancer biology at Weil Cornell Medicine. Prior studies have linked chromosomal instability to metastasis, although the reason for the link hasn't been clear. Starting hypothesis was that chromosomal instability generates a lot of genetically different tumor cells, and that a Darwinian selection process promotes the survival of the cells capable of spreading and forming distant tumors, Dr. Bakhoum said.

When he injected chromosomally unstable tumor cells into mice, he indeed found that they were many times more likely to spread and form new tumors than tumor cells in which chromosomal instability was suppressed. That was true even though both sets of tumor cells started out genetically identical, with the same abnormal numbers of chromosomes, suggesting that chromosomal instability itself was a driver of metastasis. The researchers examined gene activity in these two sets of tumor cells. They found that those with high chromosomal instability had abnormally elevated activity stemming from more than 1,500 genes - particularly in ones involved in inflammation and response of the immune system to viral infections. These were cancer cells cultured in a dish, not in the presence of any immune cells, Dr. Bakhoum said.
Recent studies by other laboratories offered some clues: Chromosomes in unstable tumor cells can leak out of the cell nucleus where they normally reside. These mis-located chromosomes encapsulate themselves to form micronuclei in the fluid, or cytosol, in the main part of the cell outside of the main nucleus. However, micronuclei tend to rupture, releasing naked DNA into the cytosol.

Cells interpret DNA in the cytosol as a sign of an infecting virus, which typically releases its DNA in the cytoplasm when it first attacks a cell. Human cells have evolved to fight this type of viral infection by sensing naked cytosolic DNA using a molecular machine called the cGAS-STING pathway. Once activated, this pathway triggers an inflammatory antiviral program. Lowering cGAS-STING levels reduced inflammation and prevented the ability of otherwise aggressive tumor cells to metastasize when injected into mice.

In an ordinary cell, an antiviral response stimulated by DNA leakage from the nucleus would soon bring about the cell's death. The researchers found, however, that tumor cells have succeded in suppressing the lethal elements of the cGAS-STING response. At the same time, they use other parts of the response to enable themselves to detach from the tumor and become mobile within the organism.

They start as in they were certain kinds of immune cells masking, which are normally activated by infection. In response, they move to the site of infection or injury in the body very quickly. By doing so, cancer cells engage in some form of lethal immune mimicry i. e. masking, and this Darwinian selection process metastasize into the social dynamics on the macroscopic level.

The evidence is based on recent studies of metastatic tumor properties, that about half of human metastases originate and expand this way. Researchers are currently investigating strategies for blocking the process.

It might not be feasible to target chromosomal instability itself, since tumor cells are inherently prone to that. Chromosomally unstable tumor cells, with their cytosolic DNA, are basically full of their own poison. Undoing their ability to suppress normal and lethal antiviral response to cytosolic DNA would, in principle, kill these aggressive cancer cells swiftly, with minimal effects on other cells. The next step is to understand better how these cells alter the normal response and how it is possible to restore it. (Bakhoum et al., 2018) Cancer cells often metastasize by hitching a ride on platelets.

\section{The reptilian brain programmes stereotyped behaviors of bio-robots}

The damages wrought by individual expansion, proliferation for selfish motives are significant compared to the Holocausts resulting from self-transcending devotion to collectively shared 
belief-systems. We have seen that the cause underlying these pathological manifestations is the reduction of the higher intelligence's influence in the brain to the primitive belief systems of the reptilian brain. More generally, it is an insufficient coordination between the emotive and discriminative faculties of the mind. When we fail to the old paranoid beliefs, combined with "fittest's" new power of destruction, this must sooner or later lead to genosuicide. The adjectival form of the term "paranoia" as it is used usually refers to a decreased mental condition characterized by false beliefs which predispose to delusions of persecution or grandeur.

Given the seed of suspicion, the human mind is capable of developing any kind of paranoid hybrid war. Human's brain of oldest heritage is basically reptilian. It forms the matrix of the brain stem and comprises much of the reticular system, midbrain and basal ganglia. The reptilian brain is characterized by greatly enlarged striatal structures which resemble the caudateputamen-globus pallidus complex of mammals. The reptilian and the old and new mammalian brains might be regarded as biological computers, each with own subjective, Gnostic, timemeasuring, memory, motor and other functions.

The reptilian brain programmes stereotyped behaviors according to instructions based on ancestral learning and ancestral memories. It seems to play a primary role in instinctually determined functions such as establishing territory, political parties' activities, finding shelter, hunting, homing, mating, breeding, imprimting, forming social hierarchies, selecting leaders, and the like. For an instinctual form of behavior is a mere phantom sufficient to trigger the entire copulatory act.

The reptilian brain seems to be a slave to precedent: see the international diplomacy. The reptilian brain determine humans obeisance to precedent in ceremonial rituals, legal actions, political persuasions, and religious convictions.

In stereotyped and repetitious forms of behavior it would seem the reptialian brain were neurosis-bound by an ancestral superego, i. e. for reduction to the bio-robotic behavior. At all events it appears to have inadequate machinery for learning to cope with new situations. In all mammals most of the primitive cortex is found in a large convolution with the limbic lobe because it surrounds the brain stem. It has become evident from clinical and experimental findings that the functions of this brain are far more encompassing, playing an important role in emotional, viscera-somatic, and endocrine functions.

The limbic cortex has similar features in all mammals and is structurally primitive compared with neocortex, what is showing its bio-robotic functions. It continues to function at an animalistic level in humans as in animals. Also in marked contrast to the neocortex, it has strong connections with the hypothalamus which plays a basic role in integrating emotional expression and viscera-somatic behavior.
Pathways linking of the three main subdivisions of the limbic system:

i. The medial forebrain bundle connects the ring of limbic cortex with the hypothalamus and other parts of the brain stem.

ii. The lower part of the ring fed by the amygdala primarily concerned with affects and behavior that insure self-preservation. Its circuits are kept busy with the selfish demands of feeding, fighting and self-protection. Structures in the upper part of the ring are connected by the septal pathway involved in expressive and feeling states that are conductive to sociability, procreation, and preservation of the species.

iii. The third pathway bypasses the olfactory apparatus, connects the hypothalamus with the anterior thalamic nuclei and the cingulated gyrus in the upper part of the ring. It also articulates with the medial dorsal nucleus which projects to the prefrontal neocortex involved in anticipation.

The limbic cortex is implicated in the generation of affective states. The mentation of limbic system would appear to involve a process whereby information is encoded in terms of affective feelings that influence its decisions and course of action. The limbic system contributes to a sense of personal identity by integrating internally and externally derived experience. With epileptic discharges arising in or near the limbic cortex of the insula, hippocampal gyrus and hippocampal formation, patients may experience illusions, alterations of perceptions, or hallucinations involving any one of the sensory systems. The parahippocampal cortex transmits impulses to the hippocampus which in turn projects to the hypothalamus and other structures of the brain stem involved in emotional, endocrine, and somatovisceral functions. The opportunity exists in the antero-medial thalamus for reciprocity of action between the third subdivision of the limbic system and prefrontal cortex.

Through these various vertical lines of communication, there is presumably the machinery for affects to arouse thoughts and for thoughts to generate affects. It may be inferred that the limbic cortex is too primitive in structure to allow communication in verbal terms. It is a problem of neocortex to interpret the feelings of the old brain. This hold also for human societies in which the medicine man, the religious reformer, and moreover the political leader is the professional who interpret and give articulation to group feelings (for example see U. S. President Donald Trump, Brexit, etc.).

Here the question of sanity does not arise because the group shares a feeling, which unlike that of the psychotic individual, lends itself to a collective belief-system. At such times political leaders step in and attempt to explain to individuals what they cannot see for themselves. The explanations and recommendations were many times in history so psychotic 
in nature as to arouse wonder in the rest of the world how civilized people could subscribe to such barbarism and carry out the dictates of its leaders (for new example see the dictate of an antihuman, bio-robotic policy of Hungarian leaders against migrants, even doing this despite the policy of the United Nations, etc.).

How influential is the reptilian brain of humans in their transformation to bio-robots during selecting and following the leaders ? It is possible that this brain in conjunction with the poorly discriminating limbic brain of bio-robots mistakes the caricature of a leader for a genuine leader. Particularly deceptive it seems, the bold, aggressive "qualities" of the psychopath that make it possible for him to put on a big show.

Recording brain activity via electrodes placed directly on the cortical surface (ECoG) provides much clearer views how the brain prepares to respond very early, even before we know how we will respond. The quicker the brain handoff, the faster people responded. The researchers found that the brain began to prepare the motor areas to respond very early, during initial stimulus presentation, suggesting that we get ready to respond even before we know what the response will be. This might explain why people often say things before they think (see the changing popularity of the Brexit, etc.).

Above evidence from brain research show that unconscious process of responding is quickly orchestrated by limbic brain and lead to the emotional, stereotyped, primitive responding to the political questions like the Hungarian bio-robots (for example Hungarian Prime Minister Victor Orbán on migration in European Union, etc.).

Big problem is that evolution by Darwinian selection process led to the production of the bio-robots in which central role is playing primitive limbic brain of the stereotyped behavior. Moreover, it has re-programmed most of the living organisms, including also humans, to a drug-dependence, endogenous (euphoric states based on the internal dopamine, endorphins) and exogenous narcomania (external euphorias based on artificial chemical drugs, narcotic plants, alcoholism). Whole above Narcotic Evolution has only made stronger the genetics of the Darwinian selection into bio-robots. These are the theoretical conclusions regarding conflict between emotion and intelligence which lead through the machinery of Darwinian selection to transformation of humans into bio-robots.

What is adaptive in the jungle is no longer adaptive in civilization. Unfortunately we still retain it and the combination of the neocortex with the reptilian brain is something that threatens to be a lethal one for the bio-robots.

\section{Narcotic Evolution means an unacceptable human conditions?}

In his First Manifesto of Surrealism we can see that throughout his adult life, what interested André Breton was less an author's work per se than the "human attitude" behind it. ...Poetry, in other words, was primarily a means of accepting the unacceptable human condition, a specific solution to the problem of our lives.

\section{Acknowledgment}

The author gratefully acknowledge the assistance of Dr. Marta Ballova, Ing. Konrad Balla, Livuska Ballova and Ing. Jozef Balla.

\section{References}

1. Bakhoum SF, Ngo B, Laughney AM, Cavallo JA, Murphy CJ, et al. (2018) Chromosomal instability drives metastasis through a cytosolic DNA response. Nature 553(7689):0020467-472.

2. M. Bartels: Newsweek, Jan. 5, 2018

3. Carhart-Harris RL, Friston KJ (2010) The default-mode, ego-functions and free-energy: a neurobiological account of Freudians ideas. Brain 133: $1265-1283$.

4. Corlett PR, Fletcher PC (2012) The neurobiology of schizotypy: Frontostriatal prediction error signal correlates with delusion-like beliefs in healthy people. Neuropsychologia 50: 3612-3620.

5. Darimont CT, Fox CH, Bryan HM, Reimchen TE (2015) The unique ecology of human predators. Science 349: 858.

6. Demaria S, Pikarsky E, Karin M, Coussens LM, Chen YC, et al. (2010) Cancer and Inflammation: Promise for Biologic Therapy. J Immunother 33: 335-351.

7. Gholipour B. We Have A Force Field Around Our Bodies An Here's How You Can Feel It. We don't end where our bodies end. The Hiffington Post.

8. Gold JI, Shadlen MN (2007) The Neural Basis of Decision Making. Annu Rev Neurosci 30: 535-574.

9. Gould SJ (1994) Scientific American.p. 84

10. Hanahan D, Weinberg RA (2011) Hallmarks of Cancer: The Next Generation. Cell 144: 646-674.

11. Haller M (2017) Persistent neuronal activity in human prefrontal cortex links perception and action. Nature Human Behaviour 2(1): 8091.

12. Hsu M, Kraibich I, Zhao C, Camerer CF (2009) Neural Response to Reward Anticipation Under Risk Is Nonlinear in Probabilities. The Journal of Neuroscience 29(7): 2231-2237.

13. Koch Ch (2004) The Quest for Consciousness: A Neurobiological Approach. Roberts and Company.

14. Kourtidis A, Ngok SP, Anastasiadis PZ (2015) Distinct E-cadherinbased complexes regulate cell behavior through miRNA processing or Src and p120 catenin activity. Nature Cell Biology 17: 1145-1157.

15. Laing RD (1960) The Divided Self: An Existential Study in Sanity and Madness. Tavistock.

16. Lin J, Marcolli M, Ooguri H, Stoica B (2014) Tomography from Entanglement.

17. https://edition.cnn.com/2018/03/31/opinions/matter-antimatterneutrinos-opinion-lincoln/index.html/

18. www.sciencedaily.com/2015/08/150824064916.htm

19. Paulus MP and Frank LR (2006) Anterior cingulated activity modulates nonlinear decision weight function of uncertain prospects. Neuroimage 30: 668-677. 
20. Prigogine I (1997) The End of Certainty. Time, Chaos, and the New Laws of Nature. First Free Press Edition, New York, pp. 161-162.

21. Raichle ME, MacLeod AM, Snyder AZ, Powers WJ, Gusnard DA, et al. (2001) A default mode brain function. Proc Natl Acad Sci USA 98: 676682.

22. Ramachandran VS, Hubbard EM (2001) Psychological Investigation into the Neural Basis of Synesthesia. Proceedings of the Royal Society of London 268: 979-983.

23. https://www.yahoo.com/news/majorana-fermion-going-changeworld-185818775.htm

24. Randall J, Tyler M (2015) Translational Medicine definition by the European Society for Translational Medicine. New Horizons in Translational Medicine 2(3): 86-88.

25. https://www.forbes.com/sites/startswithabang/2018/01/10/ new-dark-matter-physics-could-solve-the-expanding-univrsecontroversy/\#5
26. Skopec I (2017) An Explanation of Biblic Radiation: Plasma. Journal of Psychiatry and Cognitive Behavior.

27. Skopec R (2018) Artificial hurricanes and other new Weapons of Mass Destruction. International Journal of Scientific Research and Management 2(4): 297-308.

28. Skopec R (2015) Intelligent Evolution, Complexity and SelfOrganization. NeuroQuantology 13: 299-303.

29. Skopec R (2016) Translational Biomedicine and Dichotomous Correlations of Masking. Translational Biomedicine 7(1): 47.

30. Sternberg RJ (2010) Lighting the creative spark. Nature 468: 170-171.

31. Recording a thought's fleeting trip through the brain: Electrodes on brain surface provide best view yet of prefrontal cortex coordinating response to stimuli. University of California - Berkeley.

32. www.sciencedaily.com/releases/2018/01/180117114924.htm

Your next submission with Juniper Publishers
will reach you the below assets
- Quality Editorial service
- Swift Peer Review
- Reprints availability
- E-prints Service
- Manuscript Podcast for convenient understanding
- Global attainment for your research
- Manuscript accessibility in different formats
( Pdf, E-pub, Full Text, Audio)
- Unceasing customer service
Track the below URL for one-step submission
https://juniperpublishers.com/online-submission.php

